United Nations Educational Scientific and Cultural Organization and International Atomic Energy Agency THE ABDUS SALAM INTERNATIONAL CENTRE FOR THEORETICAL PHYSICS

\title{
A FERROMAGNETIC GROUND STATE FOR Mn-Co SURFACE ORDERED ALLOY ON Co(001) SUBSTRATE
}

\author{
B. M'Passi-Mabiala* \\ Département de Physique, Université Marien NGouabi, \\ Laboratoire des Materiaux et Energies, BP 69, Brazzaville, Congo \\ and
}

The Abdus Salam International Centre for Theoretical Physics, Trieste, Italy,

S. Meza-Aguilar

Escuela de Ciencias Fisico Matemáticas, Universidad Autónoma de Sinaloa, Bldv. de las Americas y Universitarios, Ciudad Universitaria, Culiacán Sinaloa, CP 80010, México and

The Abdus Salam International Centre for Theoretical Physics, Trieste, Italy

and

C. Demangeat

Institut de Physique et Chimie des Matériaux de Strasbourg, 23 rue du Loess F-63037 Strasbourg, France.

MIRAMARE - TRIESTE

July 2001

${ }^{*}$ Regular Associate of the Abdus Salam ICTP. 


\begin{abstract}
Recent Low-energy electron diffraction experiments concerning submonolayer Mn coverage on $\mathrm{Co} / \mathrm{Cu}(001)$ substrates displayed a well-defined $\mathrm{Mn}_{0.5} \mathrm{Co}_{0.5}$ surface ordered alloy. Through the Magneto-optic Kerr effect and X-ray magnetic circular dichroism a ferromagnetic coupling between $\mathrm{Mn}$ and Co was obtained. Ab initio density functional theory within generalized gradient approximation is able to explain these results.
\end{abstract}


Since the work of $\mathrm{O}^{\prime} B r i e n$ and Tonner ${ }^{1,2}$ it is well known that the magnetic moments of the ultrathin $\mathrm{Mn}$ layers grown on-top on the $\mathrm{Co} / \mathrm{Cu}(001)$ substrates is coupled ferromagnetically to Co. However, Noguera et $\mathrm{al}^{3}$, using a tight-binding Hamiltonian, were unable to obtain this ferromagnetic coupling between $\mathrm{Mn}$ and Co for a perfect $\mathrm{Mn}$ monolayer epitaxially grown on $\mathrm{Co}(001)$. Later on both Choi et $\mathrm{al}^{4}$ and O'Brien and Tonner ${ }^{5}$ within careful Low-energy electron diffraction (LEED), Magneto-optic Kerr effect (MOKE) and X-ray magnetic circular dichroism (XMCD) were able to confirm the Mn-Co ferromagnetic coupling. The discrepancy between experimental and theoretical investigations was clearly related to the appearance of a well defined Mn-Co surface ordered alloy depicted via LEED. Recent Tight-binding Linear-Muffin-Tin Orbital (TB-LMTO) calculations within the Local Density Approximation (LDA) by Meza et $\mathrm{al}^{6}$ have shown that, indeed, the surface ordered $\mathrm{Mn}_{0.5} \mathrm{Co}_{0.5}$ alloy is more stable than the perfect $\mathrm{Mn}$ monolayer on $\mathrm{Co}(001)$. Moreover a ferromagnetic configuration was one of the magnetic configurations obtained within this LDA approach. However, this solution was found marginally unstable as compared to the solution presenting an antiferromagnetic coupling. This discrepancy may originate from the effect of Oxygen on $\mathrm{Mn}^{5,7}$. However, as shown by Yoshiki et $\mathrm{al}^{7}$, the antiferromagnetic coupling between $\mathrm{Mn}$ and Co is clearly linked to the presence of Oxygen. Without Oxygen, the Mn-Co coupling is clearly ferromagnetic at odd with the recent results of Meza et al ${ }^{6}$.

From the point of view of its structural and magnetic properties, Mn can be considered as the most complex of all metallic elements ${ }^{8}$ and simple density functional theory (DFT) is of no use in this case. Hobbs and Hafner ${ }^{8}$ have investigated all known polymorphs of Mn using the generalized spin-density functional theory based on the unconstrained vector-field description of the magnetization density. Also Hoshino et $\mathrm{al}^{9}$ have depicted magnetic energy anomalies for Mn impurities in noble metals. It is therefore clear that a simple LDA approach will be most probably unable to explain the experimental results. Following the work of Blügel ${ }^{10}$ where it was shown that the formation energy for the $\mathrm{Mn} / \mathrm{Cu}$ alloy changes sign when going from LDA to Generalized Gradient Approximation (GGA), we have used here the GGA approaches of Langreth et al ${ }^{11}$ and Perdew et $\mathrm{al}^{12}$. The supercell used has been described by Meza et $\mathrm{al}^{6}$ whereas Izquierdo and Demangeat ${ }^{13}$ have discussed the suitability of the Langreth-Mehl-Hu functional. Thus the description of the theoretical model is restricted to the minimum.

We have used a scalar-relativistic version of the $k$-space TB-LMTO method ${ }^{14}$ with atomic sphere approximation. This method is based on the density functional theory ${ }^{15}$. 
In this approach we have first determinated the lattice parameter for fcc Co bulk by energy minimization of the total energy using different functionals, the von Barth-Hedin ${ }^{16}$ (LSDA-vBH), Langreth-Mehl-Hu ${ }^{11}$ (GGA-LMH) and Perdew et al ${ }^{12}$ (GGA-PW91), and the different values obtained are shown in the Table I. In this work we have used the GGA-PW91 functionals. The GGA-PW91 is known to reproduce correctly the magnetic properties of Mn as shown by Asada and Terakura ${ }^{17}$.

The overlayer-surface is modeled using the repeated slab geometry ${ }^{18}$. We take nine metallic layers separated by five layers of empty spheres (cf Fig. 1 of Meza et $\mathrm{al}^{6}$ ). This is sufficient to prevent interaction between the slabs ${ }^{19}$, which is controlled through vanishing dispersion in the direction perpendicular to the slab, and the vanishing charge in the central layer of the empty spheres. The calculations are performed using an increasing number of $k$ points, until final convergence is obtained for at least $338 k$ points in the irreducible Brillouin zone (IBZ). We restrict ourselves to two inequivalent atoms per layer.

We report here the results of calculations done on the Mn monolayer and the $2 \mathrm{D} \mathrm{Mn}_{0.5} \mathrm{Co}_{0.5}$ surface alloy on fcc $\mathrm{Co}(001)$ substrate in the nonmagnetic as well as in the magnetic cases. We have calculated the formation energy $E$ (cf. Table IV) following the Blügel formula ${ }^{10}$ :

$$
E=E_{M n-C o / C o(001)}-1 / 2\left(E_{C o / C o(001)}+E_{M n / C o(001)}\right)
$$

where $E_{M n-C o / C o(001)}, E_{C o / C o(001)}$ and $E_{M n / C o(001)}$ are the total energies of the Mn-Co surface alloy on $\mathrm{Co}(001), \mathrm{Co}(001)$ slab and $\mathrm{Mn}$ monolayer on $\mathrm{Co}(001)$ substrate, respectively.

For the $\mathrm{Mn}$ monolayer on $\mathrm{Co}(001)$ the following magnetic configurations have been considered as input: $\mathrm{p}(1 \times 1) \uparrow, \mathrm{p}(1 \times 1) \downarrow$ and $\mathrm{c}(2 \times 2)$. The converged configurations obtained (cf. Table II) show that the antiferromagnetic $\mathrm{c}(2 \times 2)$ remains in the ground state in agreement with LSDA results $^{6}$ and in disagreement with experimental results ${ }^{1,2}$.

Calculations done on nonmagnetic Mn monolayer and 2D $\mathrm{Mn}_{0.5} \mathrm{Co}_{0.5}$ surface alloy on $\mathrm{Co}(001)$ have shown that the surface alloy is more stable than the Mn monolayer. A formation energy of $5.86 \mathrm{mRy}$ has been found. Following this trend, which is in agreement with the experimental results of Choi et $\mathrm{al}^{4}$, we have investigated the magnetic case of the surface alloy. We have considered the case of $2 \mathrm{D} \mathrm{Mn}_{0.5} \mathrm{Co}_{0.5}$ surface ordered alloy (1ML-thick) in the surface plane. This has been done in relation with the results of Choi et $\mathrm{al}^{4}$ displaying surface alloy for Mn concentrations from 0.3 to 0.8 equivalent to ML. Four types of magnetic configurations ( $\mathrm{Mn} \downarrow \mathrm{Co} \downarrow, \mathrm{Mn} \uparrow \mathrm{Co} \downarrow, \mathrm{Mn} \uparrow \mathrm{Co} \uparrow$ and 
$\mathrm{Mn} \downarrow \mathrm{Co} \uparrow)$ corresponding to all possible directions of magnetization of $\mathrm{Mn}$ and Co surface atoms have been considered. Only two configurations ( $\mathrm{Mn} \downarrow \mathrm{Co} \uparrow$ and $\mathrm{Mn} \uparrow \mathrm{Co} \uparrow$ ) remain after the convergence (Table III), namely the one where the Mn atom is coupled antiferromagneticaly with the Co surface atom and the other with ferromagnetic coupling as in Meza's work ${ }^{6}$. The configuration with ferromagnetic coupling between the Mn surface atoms and the Co atoms is the ground state in agreement with the experimental results ${ }^{4}$.

The formation energy in the magnetic calculations, reported in the Table IV, is in agreement with the trends found in the nonmagnetic case i.e. a stabilization of the $\mathrm{Mn}_{0.5} \mathrm{Co}_{0.5}$ configuration. The total energies of all the magnetic configurations reported here are lower than the corresponding nonmagnetic one. However it is worthy to point out that contrary to the results reported within LDA by Meza et $\mathrm{al}^{6}$ where the formation energy decreases when going from nonmagnetic case to the magnetic one, here, within GGA the opposite is true.

We have found, within GGA, that the $\mathrm{c}(2 \times 2)$ antiferromagnetic coupling between the Mn surface atoms for the $\mathrm{Mn}$ monolayer on fcc $\mathrm{Co}(001)$ remains in the ground state in agreement with Meza et $\mathrm{al}^{6}$ theoretical results but in disagreement with the experiments. Total energy obtained within spin-polarisation calculations as a function of the magnetic configurations performed on the Mn monolayer and the $2 \mathrm{D}$ ordered $\mathrm{Mn}_{0.5} \mathrm{Co}_{0.5}$ surface alloy on $\mathrm{Co}(001)$ using TB-LMTO-ASA in the GGA-PW91 approximation have shown that, as well as in previous LSDA calculations ${ }^{6}$, the $2 \mathrm{D}$ ordered $\mathrm{Mn}_{0.5} \mathrm{Co}_{0.5}$ surface ordered alloy is the ground state configuration. A ferromagnetic ground state is however found within GGA for the $2 \mathrm{D}$ ordered $\mathrm{Mn}_{0.5} \mathrm{Co}_{0.5}$ surface alloy in agreement with experimental results ${ }^{4}$.

\section{ACKNOWLEDGMENTS}

B. M'Passi-Mabiala and S. Meza-Aguilar wish to acknowledge the Abdus Salam International Centre for Theoretical Physics (ICTP) and Swedish International Development Cooperation Agency (SIDA) for the financial support. Many thanks to J.A.C. Bland who pushes us to get a ferromagnetic ground state in agreement with his MOKE results.

This work was done within the framework of the Associateship Scheme of the Abdus Salam ICTP, Trieste, Italy. 


\section{REFERENCES}

1 W. L. O'Brien, and B. P. Tonner, Phys. Rev. B 50, 2963 (1994).

2 W. L. O’Brien, and B. P. Tonner, Phys. Rev. B 51, 617 (1995).

3 A. Noguera, S. Bouarab, A. Mokrani, C. Demangeat and H. Dreyssé, J. Magn. Magn. Mater. 156, $21(1996)$

4 B.-Ch. Choi, J. P. Bode, and J. A. C. Bland, Phys. Rev. B 58, 5166 (1998); B.-Ch. Choi, J. P. Bode, and J. A. C. Bland, J. Appl. Phys. 85, 5063 (1999).

5 W. L. O’Brien, and B. P. Tonner, Phys. Rev. B 58, 3191 (1998).

6 S. Meza Aguilar, O. Elmouhsinne, H. Dreyssé, C. Demangeat, Phys. Rev. B 63, 64421 (2001).

7 Yoshiki Yonamoto, Toshihiko Yokoyama, Kenta Amemiya, Daiju Matsumura, and Toshiaki Ohta, Phys. Rev. B 63, 214406 (2001).

8 D. Hobbs and J. Hafner, J. Phys. Condens. Matter 13, L681 (2001).

9 T. Hoshino, R. Zeller, P. H. Dederichs and M. Weinert, Europhys. Lett. 24, 495 (1993).

10 S. Blügel, Appl. Phys. A Mater. Sci. Process. A63 595 (1996).

11 D. C. Langreth and M. J. Mehl, Phys. Rev Lett. 47, 446 (1981); C.D. Hu and D.C. Langreth, Phys. Scr. 32, 391 (1985).

12 J.P. Perdew, Y. Wang, and E. Engel, Phys. Rev. Lett. 66, 508 (1991).

13 J. Izquierdo and C. Demangeat, Phys. Rev. B 62, 12287 (2000).

14 O. K. Andersen and O. Jepsen, Phys. Rev. Lett 53, 2571 (1984); O.K. Andersen, Z. Pawlowska, and O. Jepsen, Phys. Rev. B 34, 5253 (1986). The standard TB-LMTO-ASA code (version 47) developed by O.K. Andersen et al at M.P.I. Stuttgart, Germany, was used.

15 P. Hohenberg and W. Kohn, Phys. Rev. 136, B864 (1964); W. Kohn and L. J. Sham, Phys. Rev. 140, A1133 (1965).

16 U. von Barth and L. Hedin, J. Phys. C 51629 (1972).

17 T. Asada, K. Terakura, Phys. Rev. B 47, 15992 (1993).

18 M. A. Khan, J. Phys. Soc. Jpn. 62, 1682 (1993).

19 O. Elmouhssine, Ph.D Thesis, Strasbourg, France, 1998. 
TABLE I: Lattice parameter (in a.u.) and magnetic moments of Co atom in $\left(\mu_{B}\right)$ for the differents approximations.

\begin{tabular}{lrr} 
Approximation & \multicolumn{2}{c}{ Lattice magnetic } \\
& parameter & moment \\
LSDA-vBH & 6.54 & 1.56 \\
GGA-LMH & 6.64 & 1.64 \\
GGA-PW91 & 6.81 & 1.69
\end{tabular}

TABLE II: Magnetic moments (in $\mu_{B}$ ) for $\mathrm{Mn} / \mathrm{Co}(001)$ and differences of total energies per cell (in mRy) with the Perdew-Wang 91 approximation. The ground state is indicated by 0

\begin{tabular}{lrrr} 
Input & $\mathrm{c}(2 \times 2)$ & $\mathrm{p}(1 \times 1) \uparrow$ & $\mathrm{p}(1 \times 1) \downarrow$ \\
Energy & 0.0 & 46 & 159 \\
Atom & Moments & Moments & Moments \\
Mnb & 3.22 & 3.53 & -3.26 \\
Mna & -3.51 & 3.53 & -3.26 \\
Co4b & 1.15 & 1.55 & 1.17 \\
Co4a & 1.14 & 1.55 & 1.17 \\
Co3b & 1.75 & 1.77 & 1.74 \\
Co3a & 1.79 & 1.77 & 1.74 \\
Co2b & 1.71 & 1.70 & 1.71 \\
Co2a & 1.71 & 1.70 & 1.71 \\
Co1b & 1.71 & 1.70 & 1.70 \\
Co1a & 1.71 & 1.70 & 1.70 \\
\hline \hline
\end{tabular}


TABLE III: Magnetic moments (in $\mu_{B}$ ) for Mn-Co/Co(001) and difference of total energies per cell (in mRy) with GGA-PW-91 approximation. The ground state is indicated by 0 .

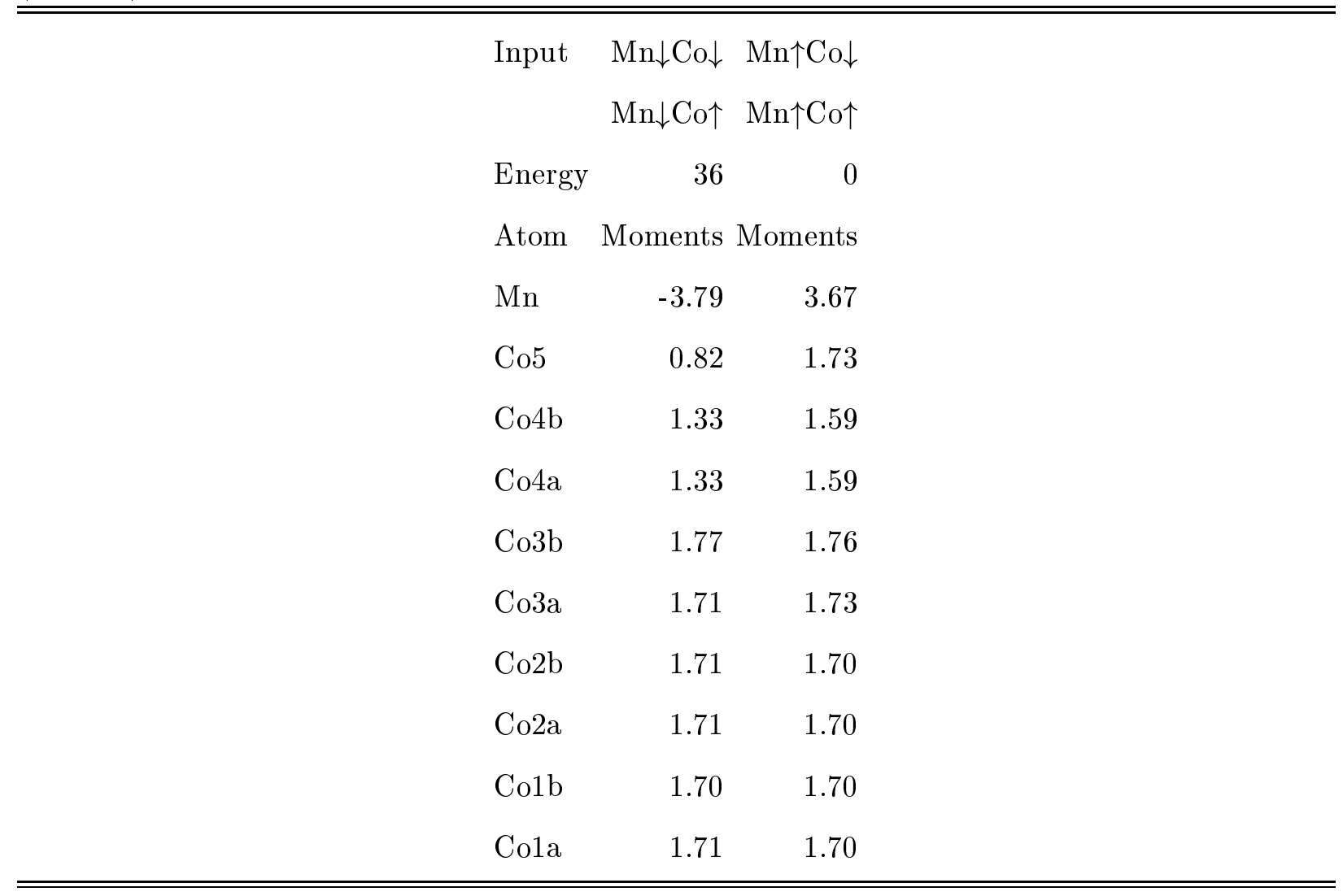

TABLE IV: Formation energies in nonmagnetic and magnetic cases with the two different approximations (in mRy).

$\begin{array}{lrr}\text { Approximation } & \text { Formation Formation } \\ & \text { Energy } & \text { Energy } \\ & \text { nonmagnetic } & \text { magnetic } \\ & -20.00 & -5.00 \\ \text { LSDA }^{6} & -5.86 & -9.77 \\ \text { GGA-PW-91 }\end{array}$

